\title{
The OTELO survey: Revealing a population of low-luminosity active star-forming galaxies at $z \sim 0.9$
}

\author{
Rocío Navarro Martínez ${ }^{1,2}$, Ana María Pérez-García ${ }^{2,1}$, Ricardo Pérez-Martínez ${ }^{3,1}$, Miguel Cerviño ${ }^{2}$, Jesús \\ Gallego $^{4}$, Ángel Bongiovanni ${ }^{5,1}$, Laia Barrufet ${ }^{6,7,8}$, Jakub Nadolny 9,10 , Bernabé Cedrés ${ }^{9,10}$, Jordi Cepa ${ }^{9,10,1}$, \\ Emilio Alfaro ${ }^{11}$, Héctor O. Castañeda ${ }^{12} \dagger$, José A. de Diego ${ }^{13,9}$, Mauro González-Otero, ${ }^{9}$, J. Jesús \\ González $^{13}$, J. Ignacio González-Serrano ${ }^{14,1}$, Maritza A. Lara-López ${ }^{15}$, Carmen P. Padilla Torres ${ }^{16,17,1}$, and \\ Miguel Sánchez-Portal ${ }^{5,1}$
}

\footnotetext{
1 Asociación Astrofísica para la Promoción de la Investigación, Instrumentación y su Desarrollo, ASPID, E-38205 La Laguna, Tenerife, Spain

2 Centro de Astrobiología (CSIC/INTA), E-28692, ESAC Campus, Villanueva de la Cañada, Madrid, Spain

3 ISDEFE for European Space Astronomy Centre (ESAC)/ESA, P.O. Box 78, E-28690, Villanueva de la Cañada, Madrid, Spain

4 Departamento de Física de la Tierra y Astrofísica, Instituto de Física de Partículas y del Cosmos, IPARCOS. Universidad

Complutense de Madrid, E-28040, Madrid, Spain

5 Instituto de Radioastronomía Milimétrica (IRAM), Av. Divina Pastora 7, Núcleo Central, E-18012 Granada, Spain

${ }^{6}$ Geneva Observatory, University of Geneva, Ch. des Maillettes 51, 1290 Versoix, Switzerland

${ }^{7}$ RAL Space, STFC Rutherford Appleton Laboratory, Didcot, Oxfordshire, OX11 0QX, UK

8 ESAC,European Space Astronomy Center, 28691 Villanueva de la Cañada, Spain

${ }^{9}$ Instituto de Astrofísica de Canarias (IAC), E-38200 La Laguna, Tenerife, Spain

${ }_{11}^{10}$ Departamento de Astrofísica, Universidad de La Laguna (ULL), E-38205 La Laguna, Tenerife, Spain

11 Instituto de Astrofísica de Andalucía, CSIC, E-18080, Granada, Spain

12 Departamento de Física, Escuela Superior de Física y Matemáticas, Instituto Politécnico Nacional, Mexico D.F., Mexico

13 Instituto de Astronomía, Universidad Nacional Autónoma de México, Apdo. Postal 70-264, 04510 Ciudad de México, Mexico

${ }^{14}$ Instituto de Física de Cantabria (CSIC-Universidad de Cantabria), E-39005 Santander, Spain

15 Armagh Observatory and Planetarium, College Hill, Armagh, BT61 DG, UK

16 INAF, Telescopio Nazionale Galileo, Apartado de Correos 565, E-38700 Santa Cruz de la Palma, Spain

${ }^{17}$ Fundación Galileo Galilei - INAF Rambla José Ana Fernández Pérez, 7, 38712 Breña Baja, Tenerife, Spain
}

Received — / Accepted —

\section{ABSTRACT}

\begin{abstract}
Aims. We study a sample of $\mathrm{H} \beta$ emission line sources at $z \sim 0.9$ to identify the star-forming galaxies sample and characterise them in terms of line luminosity, stellar mass, star formation rate, and morphology. The final aim is to obtain the $\mathrm{H} \beta$ luminosity function of the star-forming galaxies at this redshift.

Methods. We used the red tunable filter of the instrument Optical System for Imaging low Resolution Integrated Spectroscopy (OSIRIS) at Gran Telescopio de Canarias (GTC) to obtain the pseudo spectra of emission line sources in the OTELO field. From these pseudo spectra, we identified the objects with $\mathrm{H} \beta$ emission. As the resolution of the pseudo spectra allowed us to separate $\mathrm{H} \beta$ from [O III], we were able to derive the $\mathrm{H} \beta$ flux without contamination from its adjacent line. Using data from the extended OTELO catalogue, we discriminated AGNs and studied the star formation rate, the stellar mass, and the morphology of the star-forming galaxies.

Results. We find that our sample is located on the main sequence of star-forming galaxies. The sources are morphologically classified, mostly as disc-like galaxies $(76 \%)$, and $90 \%$ of the sample are low-mass galaxies $\left(M_{*}<10^{10} \mathrm{M}_{\odot}\right)$. The low-mass star-forming galaxies at $z \sim 0.9$ that were detected by OTELO present similar properties as low-mass starforming galaxies in the local universe, suggesting that these kinds of objects do not have a favorite epoch of formation and star formation enhancement from $z \sim 1$ to now. Our sample of $40 \mathrm{H} \beta$ star-forming galaxies include the faintest $\mathrm{H} \beta$ emitters detected so far. This allows us to constrain the faint end of the luminosity function for the $\mathrm{H} \beta$ line alone with a minimum luminosity of $\log L=39 \mathrm{erg} \mathrm{s}^{-1}$, which is a hundred times fainter than previous surveys. The dust-corrected OSIRIS Tunable Emission Line Object survey (OTELO) H $\beta$ luminosity function established the faint-end slope as $\alpha=-1.36 \pm 0.15$. We increased the scope of the analysis to the bright end by adding ancillary data from the literature, which was not dust-corrected in this case. The obtained slope for this extended luminosity function is $\alpha=-1.43 \pm 0.12$.
\end{abstract}

Key words. techniques: imaging spectroscopy - surveys - catalogs - galaxies: starburst - galaxies: luminosity function - galaxies: star formation - cosmology: observations

\section{Introduction}

The study of star formation along cosmic time is a key tool for characterising the evolution of galaxies and the physics involved at cosmological scales. All the processes concurring in a given space and time affect this activity and, at the same time, are reflected in how the star formation takes place.

The star formation rate (SFR hereafter) can be estimated though different indicators, from the X-ray to ra- 
dio wavelengths (see the review by Kennicutt 1998; Kennicutt \& Evans 2012), using both continuum and emission lines. The luminosity of the $\mathrm{H} \alpha$ emission line is a reliable indicator which scales linearly with the number of ionising photons produced by young massive stars (e.g. Hayashi et al. 2018; Coughlin et al. 2018; Matthee et al. 2017; Sobral et al. 2015; Fujita et al. 2003; Gallego et al. 1995). It is also the reddest (hence less affected by internal extinction) and stronger (hence with a lower relative correction due to stellar absorption) Balmer line in the optical, although formally any other Balmer line may also be used (see Cerviño et al. 2016, for a discusion about the SFR calibration). For redshifts higher than $z=0.4$, where $\mathrm{H} \alpha$ is not available in the optical range, the second best choice is $\mathrm{H} \beta$ as the second stronger recombination line. We note that there are also some collisional lines which can also be used as a SFR proxy as [O II] $\lambda 3727$ (Villaverde et al. 2010), although their calibration requires the use of photoionisation codes and it is affected by metallicity and dust attenuation.

Different emission line surveys have been carried out to measure $\mathrm{H} \alpha$ and $\mathrm{H} \beta$ spectral lines (see Newman et al. 2013; Driver et al. 2009; Geach et al. 2008; Gallego et al. 1995, among others). These surveys accurately determine the spectroscopic redshifts and therefore constrain the volume under study more accurately. In addition, emission-line surveys allow one to observe fainter galaxies due to the relatively higher brightness of the emission compared to the continuum. Slitless surveys are usually based on sets of narrow and medium or broad band observations, overcoming the need for a sample of pre-selected objects. However they provide less accurate estimations of redshift and line fluxes.

In this paper we exploit the data from OSIRIS Tunable Emission Line Object survey (OTELO) (Bongiovanni et al. 2019 , hereafter OTELO-I) to analyse the population of $\mathrm{H} \beta$ emitters at $z \sim 0.9$. OTELO is a slitless pencil beam survey that uses the Red Tunable Filter (RTF) of the OSIRIS instrument (Cepa et al. 2003) at the 10.4m Gran Telescopio Canarias (GTC, Alvarez et al. 1998), and it was designed to overcome the drawbacks of integral field surveys by a discrete scanning of the spectral range of interest along the full field of view of the instrument, producing pseudospectra with a resolution of $\mathrm{R} \sim 700$. OTELO targeted a region relatively free of sky emission lines to find emission lines sources (ELSs) at different co-moving volumes with mean redshifts of up to 6.5. The RTF was configured to scan a window of $230 \AA$ centred at $9175 \AA$ in a $7.5 \times 7.4 \operatorname{arcmin}^{2}$ area located at the south-west edge of the most deeply explored region of the Extended Groth Strip (EGS hereafter). The limiting line flux achieved is $5 \times 10^{-19} \mathrm{erg} \mathrm{s}^{-1} \mathrm{~cm}^{-2}$, which makes it the deepest emission line survey to date (see OTELO-I for a full description of the survey).

The redshift for $\mathrm{H} \beta$ emitters in our sample is centred at $z \sim 0.87$, given the wavelength coverage of the OTELO survey. The spectral resolution of the data makes it possible to deblend $\mathrm{H} \beta$ from its close emision features [O III] $\lambda 4959,5007$. This allows us to study the star formation activity at this redshift range, and, using the data in the extended OTELO catalogue, characterise these objects in terms of their photoionisation engine (either active galaxy nuclei (AGNs) or star formation activity), morphology, and stellar mass.

Being able to measure $\mathrm{H} \beta$ without contamination from [O III] $\lambda 4959,5007$ also allowed us to build the luminosity function ( $\mathrm{LF}$ hereafter) of $\mathrm{H} \beta$ emitters at this redshift.
Previous works have analysed the LF function with narrowband images that were not able to resolve the contribution from the $\mathrm{H} \beta$ line alone, hence obtaining the $\mathrm{LF}$ of $\mathrm{H} \beta+[\mathrm{O}$ III] together. Up to now, only Comparat et al. (2016) have studied a $\mathrm{LF}$ of $\mathrm{H} \beta$ in the range of $z \sim 0.8$. However, Comparat et al. (2016) only cover the bright end of the luminosity function (up to $10^{40} \mathrm{erg} / \mathrm{s}$ ), based on spectroscopic data of VVDS (Le Fèvre et al. 2013) and DEEP2 (Newman et al. 2013) surveys. We took advantage of the unprecedented depth of OTELO data to extend the $\mathrm{H} \beta$ LF faint end and constrained the number of galaxies at low line luminosities.

It is well known that the relationship between the SFR and mass for star-forming galaxies (SFGs), the so-called main sequence (MS) of star-forming (SF) galaxies (Speagle et al. 2014; Noeske et al. 2007). Galaxies on this MS formed stars at much higher rates in the distant universe than they do today. Moreover, the bulk of SF thus appears to have occurred earlier in massive galaxies compared to less massive systems (Bouché et al. 2010; Elbaz et al. 2007). Again, due to the characteristics of the OTELO survey, our sample of $\mathrm{H} \beta$ emitters are low-mass star-forming galaxies. Then, we were able to analyse the location of low-mass SFGs at $z \sim 0.9$ in the SFR-mass diagram and its impact on the evolution of star-forming galaxies.

This paper is structured as follows. In Section 2 we describe the selection of $\mathrm{H} \beta$ emitters in the OTELO survey and the obtention of the parameters that we use in our analysis. Section 3 addresses the characterisation of $\mathrm{H} \beta$ emitters. In Section 4 we present the observed LF, including the description of main biases and corrections. The discussion of the results and a comparison with similar data are presented in Section 5. Finally, Section 6 reports the conclusions of this work.

Throughout this paper, we assume a standard $\Lambda$-cold dark matter cosmology with $\Omega_{\Lambda}=0.7, \Omega_{m}=0.3$, and $\mathrm{H}_{0}=$ $70 \mathrm{~km} \mathrm{~s}^{-1} \mathrm{Mpc}^{-1}$. All magnitudes are given in the AB system.

\section{The OTELO sample of $\mathrm{H} \boldsymbol{\beta}$ emitters}

\subsection{The OTELO catalogue}

The OTELO-observed window is a region of almost 56 square arcmin in the EGS field centred at RA=14h $17 \mathrm{~m}$ $33 \mathrm{~s}$, Dec $=+52^{\circ} 28^{\prime} 22^{\prime \prime}$ (J2000.0), at 36 different wavelengths equally spaced between $9070 \AA$ and $9280 \AA$. Using OSIRIS guaranteed time, a total exposure time of 108 hours was dedicated to obtain OTELO data. A source list was extracted from the co-added image, and the flux at each wavelength was obtained and complemented with data from the CFHTLS survey (T0007 Release), Hubble Space Telescope Advanced Camera for Surveys (HST-ACS), and near-infrared (NIR) data from the WIRcam Deep Survey (WIRDS, Release T0002) to form the core catalogue. Ancillary data from X-ray, ultraviolet (UV), mid-infrared (MIR), and far-infrared (FIR) catalogues were added through educated cross match techniques developed by Pérez-Martínez (2016). These data are included in the OTELO multiwavelength catalogue.

The catalogue contains 11237 raw entries with up to 24 photometric detections each. The spectral energy distributions (SED) for these were obtained with LePhare (Arnouts et al. 1999; Ilbert et al. 2006), using a galaxy template library with the four standard Hubble types (Coleman et al. 1980) and six SF galaxy templates (Kinney et al. 1996) to 
estimate photometric redshifts as described in OTELO-I. Filters used to obtain photo-z's cover form 1200 to $10000 \AA$ (NUV from GALEX; $\mathrm{u}, \mathrm{g}, \mathrm{r}, \mathrm{i}$, and $\mathrm{z}$ from CFHTLS; and $\mathrm{J}, \mathrm{H}$, and Ks from WIRDS). The extinction law of Calzetti et al. (2000) was adopted, with values of extinction $\mathrm{E}(\mathrm{B}-\mathrm{V})$ ranging from 0 to 1.1 in steps of 0.05 . The value and quality of the photo- $z$ are included in the final OTELO catalogue. The selection of emitting objects candidates is based on the analysis of the pseudo-spectra. The presence of flux excess as part of a possible emission-line leads to 5322 preliminary emission line candidates. A pseudo-spectrum is the result of the convolution in the wavelength space of the input SED of a given source, with the RTF instrumental response characterised by a succession of airy profiles. For more detailed information on the building of the final OTELO catalogue, pseudo-spectra extraction, photometric redshifts estimates, and the selection of emitting objects, readers can refer to OTELO-I and Bongiovanni et al. (2020).

\section{2. $H \beta$ emitters selection}

In this work we focus on the analysis of a sample of $\mathrm{H} \beta$ emitting galaxies. To this end, we first describe the implementation of the selection steps used in this particular science case to identify those that present emission on the $\mathrm{H} \beta$ line:

Firstly, an initial sample of possible $\mathrm{H} \beta$ emitters was created by those objects falling in the redshift interval $0.85<z_{\text {phot }}<0.91$ in order to safely include all $\mathrm{H} \beta$ emission line galaxy candidates. The photo- $z$ solutions that include the OTELO-deep photometry provide 87 preliminary ELSs. From this selection, there were 38 objects with [O III] $\lambda 4959,5007$ emission, which has been previously studied in Bongiovanni et al. (2020).

Secondly, each object from this sample of $\mathrm{H} \beta$ precandidates was analysed using a web-based interactive graphic user interface (GUI) designed for the analysis of OTELO data ${ }^{1}$. This tool provides the following for each object: the pseudo-spectrum, the $z_{\text {phot }}$ solutions obtained by LePhare fitting together with the SED of the object and the LePhare solutions, the stamps in all broad band filters (including HST and OTELO-deep), and all the available information about the object in the database such as ancillary spectroscopic redshifts provided by DEEP2 of the source if available, and data included in the NASA/IPAC Extragalactic Database (NED), if catalogued. Taking into account all available data for each galaxy, we refined the obtained $z$ by eye verification of the presence of the emission line and the possible $z_{\text {phot }}$ solutions, and/or we reject the object as a $\mathrm{H} \beta$ emitter galaxy. After each pre-candidate was analysed by several collaborators following the same criteria, a final sample of true $\mathrm{H} \beta$ emitters was obtained based on the degree of confidence of the most reliable redshift value assigned by such a process, $z_{\text {GUESS }}$. We note that such a reliable redshift is usually assigned to the peak of the $\mathrm{H} \beta$ line observed in the pseudo-spectra. From this analysis, we finally identified 47 objects as $\mathrm{H} \beta$ emitters.

After this process, we obtained 47 objects classified as $\mathrm{H} \beta$ emitters by at least three collaborators. However, six objects are marked as reliable $\mathrm{H} \beta$ sources, but they present some potential problems as would be a very large uncertainty in the $z_{\text {phot }}$ solutions, such as truncated lines (three

\footnotetext{
1 http://research.iac.es/projecto/otelo
}

objects) or possible double sources (three objects). These objects, although classified as $\mathrm{H} \beta$ emitters, were not included in the analysis and flux measurements, making up a final sample of 41 objects for the analysis. Moreover, we discriminate those sources which are robust bona fide $\mathrm{H} \beta$ emitters, meaning they: (i) have a reliable photometric redshift; (ii) have a clearly defined line in the pseudo spectra; and (iii) are well defined in every band. This criteria is met by 28 sources, making up the robust $\mathrm{H} \beta$ sub-sample. The remaining 13 objects do not fulfill one of the previous terms, but they are also included as $\mathrm{H} \beta$ emitters.

\subsection{Identifying AGNs}

In order to build the luminosity function of $\mathrm{H} \beta$ emitters at $z \sim 0.9$ as well as to infer reliable fluxes, luminosity, and stellar masses, we only used recipes aimed for SFGs. Thus, we must discriminate between SFGs and active galactic nuclei (AGNs).

As explained in Section 2.1, the extended catalogue of OTELO covers from the X-ray to FIR range. First, in order to identify AGNs, we checked if any part of the $\mathrm{H} \beta$ sample has emission in soft X-rays, because the high energy emission is the most efficient method to select AGNs. However, there are not $\mathrm{X}$-ray data anywhere in the sample.

On the other hand, we used the IRAC based criteria proposed by Donley et al. (2012). This method uses the Spitzer/IRAC bands (at 3.6, 4.5, 5.8, and $8.0 \mu \mathrm{m}$ ) to define an empirical region where the AGNs are located. We found that only one source of our sample satisfied one of these criteria and was hence classified as an AGN galaxy. This low fraction of AGNs $(\sim 2 \%)$ can be explained by the fact that OTELO is a very deep survey, but covering a small volume compared with similar surveys $\left(5190 \mathrm{Mpc}^{-3}\right.$ for our $\mathrm{H} \beta$ sample, see Section 4.1). Moreover, we note that only ten objects of our $\mathrm{H} \beta$ emitters have IRAC counterparts $(\sim 25 \%$ of the sample) and, therefore, the fraction of AGNs could be $\sim 8 \%$. Finally, due to the low line luminosity of our $\mathrm{H} \beta$ emitters, as we previously noted, we do not expect a noticeable AGN fraction despite the redshift window explored and the AGN fraction depends more on luminosity than redshift (Chiang et al. 2019). Even so, the obtained fraction of AGNs is consistent with the work of RamónPérez et al. (2019b) who found an AGN fraction of about $7 \%$ for the $\mathrm{H} \alpha$ sample of OTELO at $z \sim 0.4$. Bongiorno et al. (2010) find a $5 \%$ of type-2 AGNs (from a narrow-line selection) at $z \sim 0.8$ for a sample of $1620[\mathrm{O} \mathrm{III]}] \mathrm{H} \beta$ emitters, which is also consistent with our result.

\subsection{H $\beta$ fluxes and luminosities}

The pseudo-spectra of the remaining $40 \mathrm{H} \beta$ SFG emitters where the flux can be obtained were analysed in a similar way as described in Nadolny et al. (2020) for the case of $\mathrm{H} \alpha$ sources, based in an inverse deconvolution process of the pseudo spectrum. In summary, we obtain the isophotal flux measured in individual frames as the best approximation for a corrected aperture flux in crowded fields, avoiding then aperture losses. The flux calibration is done using field stars, and it is consistent with the flux density obtained with the SDSS-DR12 photometry (see OTELO-I for details). Then we assume a model spectra as a rest-frame spectra defined by Gaussian profiles of the $\mathrm{H} \beta$ line defined by its amplitude 

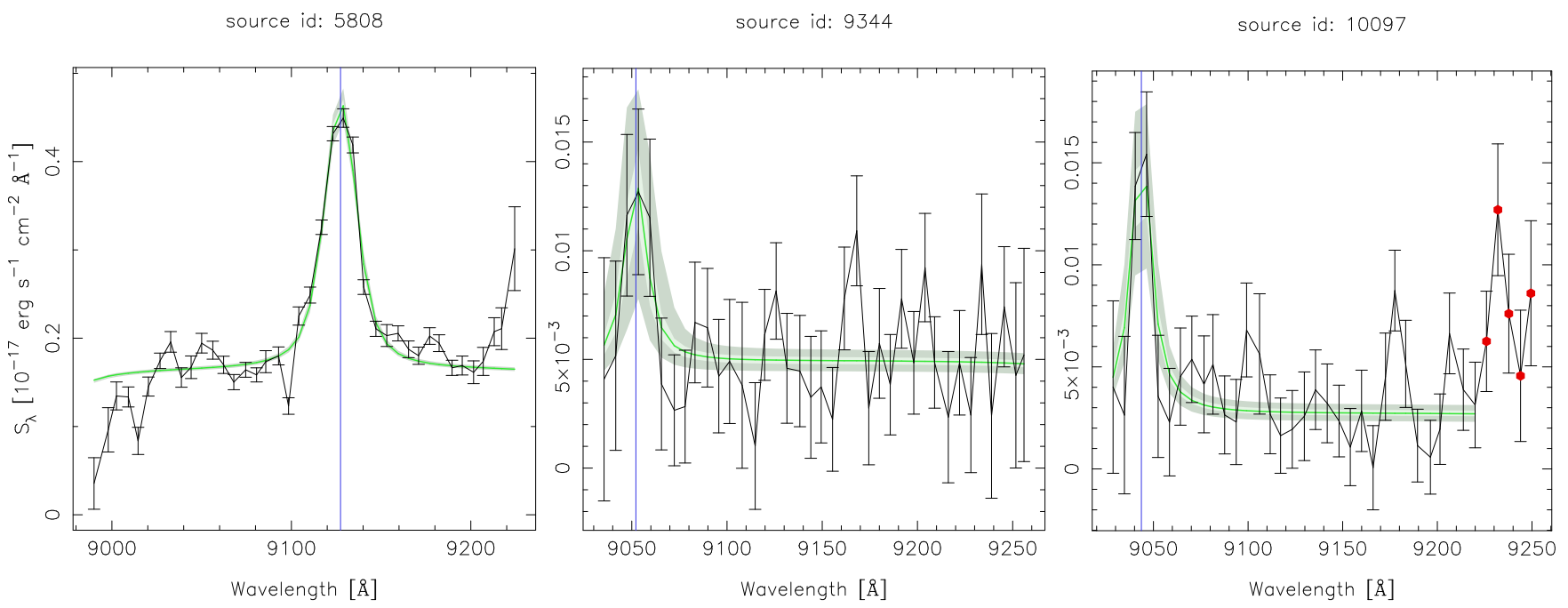

Fig. 1. Examples of pseudo-spectra. In each plot, the black line with error bars represents the observed pseudo-spectrum, the green line shows the best fitted result of the deconvolution process, and the light green and light grey shadow area shows the envelope of solutions from the deconvolution process including $25 \%$ and $68 \%$ of the solutions, respectively. The blue vertical line marks the observed $\mathrm{H} \beta$ wavelength. The left plot (id:5808) shows a pseudo-spectrum with a good signal and good fit; the middle plot shows the source id:9344, which has a low signal to noise, and therefore a larger uncertainty in the deconvolution results; the right plot shows the case of the source 10097, where some spurious points in the pseudo-spectrum were masked during the fitting process.

$f_{\mathrm{H} \beta}$ and line width $\sigma$, as well as a constant continuum level of $f_{c}$ and $f_{\text {mod }}\left(z, f_{c}, \sigma, f_{\mathrm{H} \beta}\right)$. We performed $10^{6}$ Monte Carlo simulations where we varied $z, f_{c}, \sigma$, and $f_{\mathrm{H} \beta}$ in such a way that a likelihood function of all variables was mapped. After that, we marginalised the likelihood function over each of the parameters and obtained the corresponding probability density functions (PDFs). We used the statistical mode as a reference and selected the results within the $68 \%$ confidence interval around this value. For the analysed sample, the resulting PDFs are quite symmetric, allowing, as in this case, the $68 \%$ interval to also be a good proxy for the standard deviation. All details are similar to those in Nadolny et al. (2020), except that in our case we sampled the redshift space by following a flat distribution in the range of $z_{\text {GUESS }} \pm 0.002$ instead of the 0.001 one used in that work.

In Figure 1 we show three examples of the fit obtained after the deconvolution process. We note that the pseudospectra have different qualities and signal-to-noise ratios. Such qualities are consistent with the uncertainties obtained.

It is noteworthy that the $\mathrm{H} \beta$ flux obtained from deconvolution does not take the effect of stellar absorption into account from old stellar populations. Hence, to correct the $\mathrm{H} \beta$ flux of this effect, we adopted the same prescription used by Nadolny et al. (2020), with a EW=2.5 $\AA$ corresponding to the stellar absorption based on Hopkins et al. (2003, 2013). We note that such a value is also assumed for $\mathrm{H} \alpha$ in Hopkins et al. (2013). However, these authors show that using the same value for both recombination lines is the best choice based on their simulations. The stellar absorption correction was performed for all sources in a selfconsistent way (i.e. over the entire Monte Carlo set and obtaining the resulting PDF and uncertainties). The resulting distribution of $\mathrm{EW}(\mathrm{H} \beta)$ is shown in the top left panel in Fig. 2. The minimum $\operatorname{EW}(\mathrm{H} \beta)$ is 8.9 with 39 objects with an $\mathrm{EW}(\mathrm{H} \beta)$ larger than $10 \AA$, so the choice of the stellar absorption correction has a minor impact on our estimates.
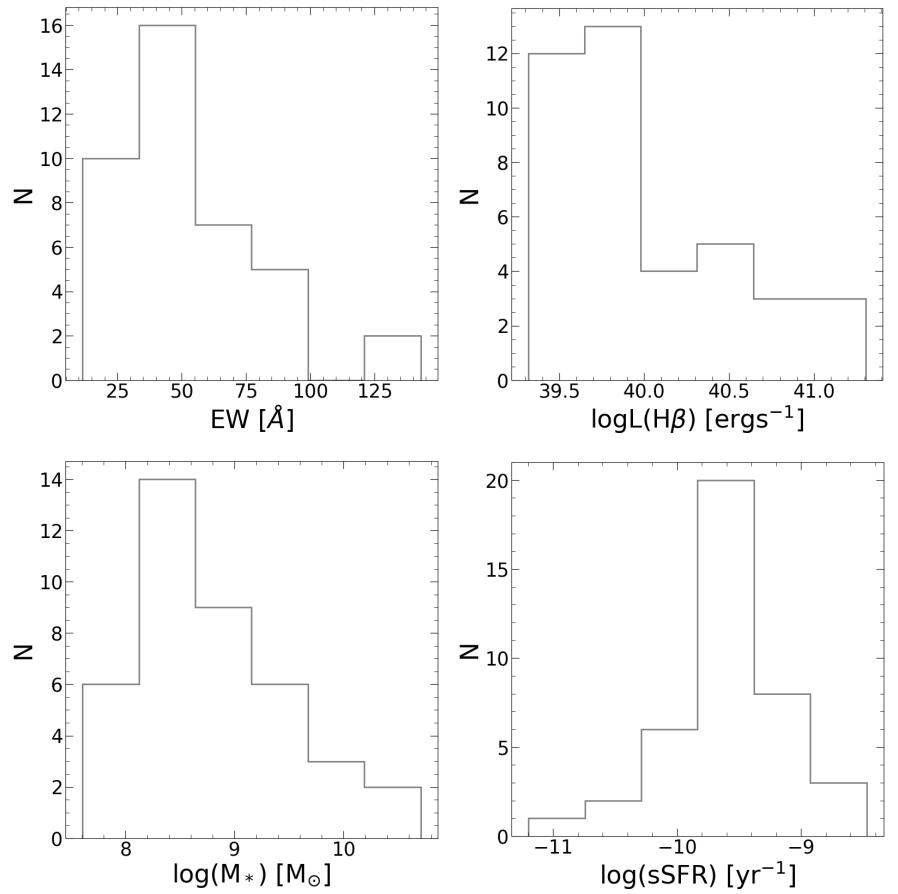

Fig. 2. Distribution of significant parameters for the $\mathrm{H} \beta$ sample. Top left: Rest-frame equivalent widths. Top right: Corrected luminosity derived from flux. Bottom left: Stellar masses. Bottom right: Specific SFR (see the text for details).

The $\mathrm{H} \beta$ fluxes were corrected for extinction by applying the law described in Cardelli et al. 1989:

$f_{\mathrm{H} \beta}^{c}=f_{\mathrm{H} \beta}^{o} \times 10^{C_{\mathrm{H} \beta}}$,

where $f_{\mathrm{H} \beta}^{c}$ is the extinction-corrected flux, $f_{\mathrm{H} \beta}^{o}$ is the estimated flux from the inverse deconvolution process and corrected for underling stellar absorption, $C_{\mathrm{H} \beta}=1.488 \times E(B-$ 
$V)$ is the $\mathrm{H} \beta$ decrement, and $E(B-V)$ are the broad-band colour excess values obtained from LePhare SED fitting, which includes the intrinsic extinction of the template if it is an SF from Kinney et al. (1996). The median value of extinction in our sample is 0.1 . Also, we looked for MIR and FIR emission in our sources from Spitzer and/or Herschel data, but only a few of the sources, eight to be exact, have detectable emission. These galaxies correspond to the most massive galaxies in the sample $\left(\log M_{*}>9.5\right)$, with $E(B-V)$ values equal or larger than 0.1 . This result is consistent with the inferred low extinction values in the sample. We are aware that by using the $\mathrm{H} \beta$ decrement, we assume a common colour excess all along the galaxy independently of its components (stellar and different gas phases) and position.

Finally, the $\mathrm{H} \beta$ luminosity of each object of our $\mathrm{H} \beta$ sample was obtained as $L(\mathrm{H} \beta)=4 \pi f_{\mathrm{H} \beta}^{c} D_{L}^{2}$, where $D_{L}$ is the luminosity distance. The observed luminosity range covered is $39.07<\log L(\mathrm{H} \beta)\left[\mathrm{erg} \mathrm{s}^{-1}\right]<40.98$ (see Figure 2).

\subsection{Stellar masses and SFR}

Stellar masses $\left(M_{*}\right)$ for the overall OTELO sample were computed from the mass-to-light ratio prescription of López-Sanjuan et al. (2019) for star-forming galaxies (more details about obtaining stellar masses in the OTELO survey can be found in Nadolny et al. (2021). The stellar mass for the $\mathrm{H} \beta$ sample is in the range of $10^{7.6}<M_{*}<10^{10.7} \mathrm{M}_{\odot}$. The resulting distribution of $\log M_{*}$ is shown in Fig. 2. We note that the distribution is concentrated at values of $M_{*}$ below $10^{9.5}$, which points towards a SF nature of the $\mathrm{H} \beta$ emission (see Sect. 2.3, instead of AGN hosts).

The SFR of our $\mathrm{H} \beta$ emitters was obtained by following the standard calibration of Kennicutt \& Evans (2012) for solar metalliciy and the initial mass function (IMF) from Kroupa (2001), but replacing $\mathrm{H} \alpha$ by $\mathrm{H} \beta$ luminosity:

$$
\mathrm{SFR}\left(\mathrm{M}_{\odot} \mathrm{yr}^{-1}\right)=5.37 \times 10^{-42} \times I(\mathrm{H} \alpha) / I(\mathrm{H} \beta) \times L(\mathrm{H} \beta)\left[\mathrm{erg} \mathrm{s}^{-1}\right],
$$

where the factor $I(\mathrm{H} \alpha) / I(\mathrm{H} \beta)=2.86$ corresponds to the ratio between these lines for solar metallicity Case B recombination and typical electron temperature and density for individual H II regions (Storey \& Hummer 1995). The specific SFR (sSFR) is subsequently estimated as SFR/ $M_{*}$. The distribution of sSFR is shown in the bottom right panel of Fig. 2 and it is studied in detail in Sect. 5.1. The catalogue of the selected sources, including their estimates for $z, \mathrm{H} \beta$ flux, EW, stellar masses, SFR, and $g-i$, is shown in the appendix in Tables A.1 (the 27 bona fide sources excluding the AGN in the sample) and A.2 (the 13 additional sources).

\section{Physical properties of the $\mathbf{H} \beta$ sample}

As we have described in the previous section, the final sample of $\mathrm{H} \beta$ emitters that we used consists of 40 objects. This sample of $\mathrm{H} \beta \mathrm{ELSs}$ is distributed in the range from $0.855 \leq z \leq 0.904$. The observed flux is lower than $2 \times 10^{-16} \mathrm{erg} \mathrm{s}^{-1} \mathrm{~cm}^{-1}$ for $83 \%$ of the sample and $73 \%$ of our sources have a rest-frame equivalent width under $60 \AA$. Now, we analyse the morphology and colour properties of the sample as a cross-check test of their star-forming nature.
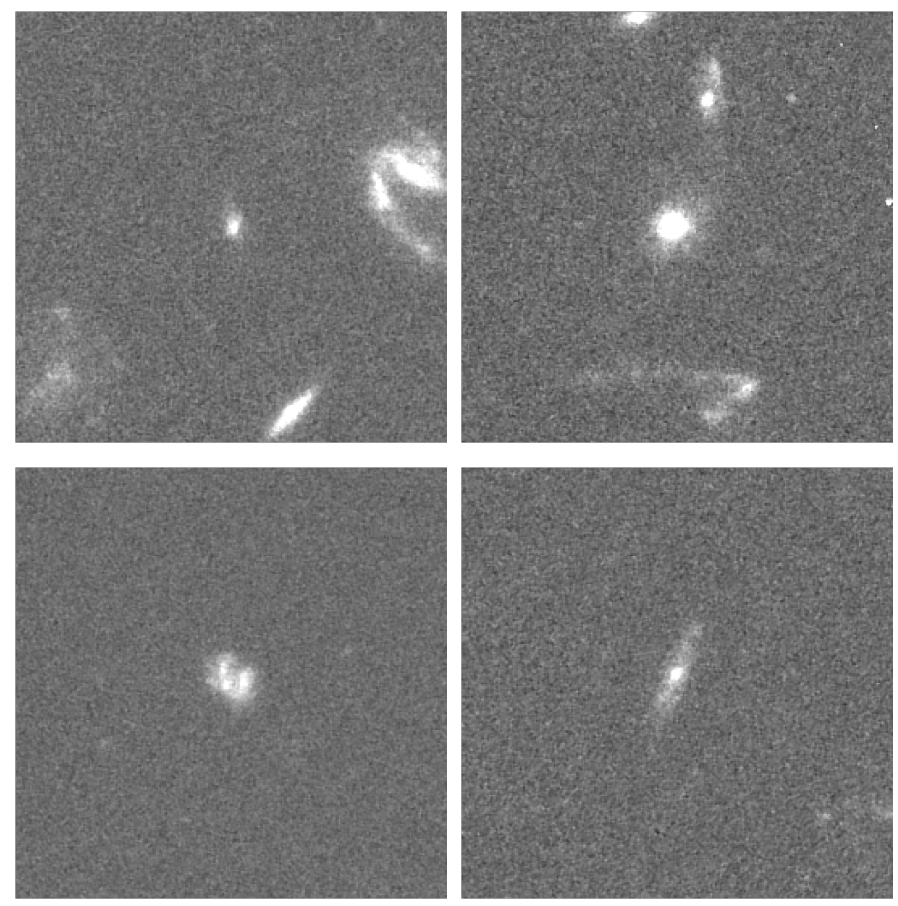

Fig. 3. HST(F814W) images as representative examples of morphology types. The angular size for all of them is $8 \operatorname{arcsec}^{2}$. Top left: Disc-like galaxy, which corresponds to the only AGN in our sample. Top right: Elliptical galaxy. Bottom left: Clumpy cluster galaxy. Bottom right: Discy galaxy.

\subsection{Morphology}

To determine the morphological features of our galaxy sample, we made use of the available ${ }^{2}$ HST high-resolution (F606W and F814W) images for a visual morphological classification. The detailed morphological analysis of all OTELO sources up to $z=2$ is in the scope of a forthcoming paper (Nadolny et al. 2021).

Our visual classification is done using MorphGUI, a graphic user interface for morphological analysis developed by CANDELS (see Kartaltepe et al. 2015). We modified the interface in order to provide additional morphological classes to extend the classical Hubble scheme to be able to take peculiarities into account (i.e. chain galaxies, tadpoles, clumpy cluster types) found at higher redshifts (Elmegreen et al. 2007). For 15 sources of the $\mathrm{H} \beta$ sample, it was not possible to assign a morphology classification. Nine of these sources are not detectable in the HST image, and the remaining six are outside the HST-ACS footprint. The EWs of these 15 objects are larger than $37 \AA$. Considering that the EW of our sample is above $8.9 \AA$ and as stated in Bongiovanni et al. (2019), the minimum value of EW detected with $p \leq 0.95$ is $5.7 \AA$ ([O III $]$ at $z=0.8$ ), we do consider these 15 objects as bona fide sources. The bulk of classified sources $(20$, about $\sim 76 \%$ ) are disc-like galaxies, two are early-type, two are clumpy clusters, and one is an interacting system. The morphological classification of the $\mathrm{H} \beta$ sample shows similar results to those from the OTELO [O III] 44959,5007 (Bongiovanni et al. 2020) sample, where again $85 \%$ of the morphologically classified galaxies are discs, with the HIZELS survey for $\mathrm{H} \alpha$ emitters at $z \sim 0.8$

2 http://aegis.ucolick.org/mosaic_page.htm 


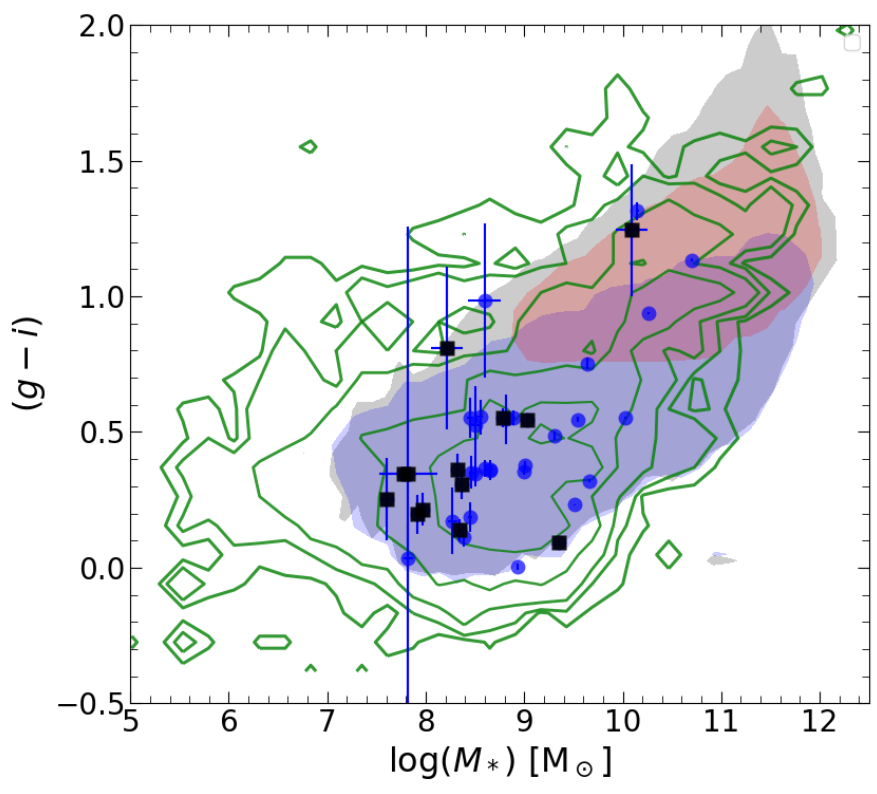

Fig. 4. Colour $-M_{*}$ diagram. Blue points show $\mathrm{H} \beta$ ELS and green contours represent the whole OTELO sample. The filled contour in grey shows the envelope of SDSS-DR7 data, while filled red and blue contours show red and blue clouds separated with the empirically estimated limit of Bluck et al. (2014). For the $\mathrm{H} \beta$ sample, we differentiate the robust sub-sample of 27 galaxies from the remaining 13 with blue circles and black squares, respectively.

$(75 \pm 8 \%$, Sobral et al. 2013) and with Villar et al. (2008) in a $\mathrm{H} \alpha$ near-infrared narrowband survey at $z=0.84$.

Figure 3 displays HST images for three different morphology types as well as the AGN host. The sources used for these three morphology examples also have DEEP2 spectra data available, which are included, and the DEEP2 spectroscopic redshift is consistent with our results. We note, however, that the AGN host was excluded from the previous analysis and it is only included here for illustrative purposes.

\subsection{Colour-mass relation}

Figure 4 shows the obtained stellar masses as a function of the rest-frame $(g-i)$ colour for the star-forming $\mathrm{H} \beta$ sample. For comparison, in the same figure, we show the SDSS overall sample together with the blue and red cloud empirical colour division from Bluck et al. (2014), obtained using data from SDSS-DR7. Following the same approach as Nadolny et al. (2020), we identified blue and red clouds using the $(g-r)$ colour and represent them in terms of $(g-i)$, only for illustrative purposes. Most of the galaxies from the $\mathrm{H} \beta$ sample are in the blue cloud, as expected, and its stellar masses are mainly in the low mass region $\left(\mathrm{M}_{*}<10^{10} \mathrm{M}_{\odot}\right)$.

Thus, in summary, our sample is mainly composed of low-mass SF galaxies. These are ideal to study the lower luminosity end of the $\mathrm{H} \beta$ luminosity function at $z \sim 0.9$ and the low mass end of the SFR properties at such a redshift.

\section{4. $\mathrm{H} \beta$ luminosity function}

Using our sample of $\mathrm{H} \beta$ star-forming galaxies, we obtain the luminosity function, $\mathrm{LF}(\mathrm{H} \beta)$, of OTELO at $z \sim 0.9$. The estimation of LF takes completeness and cosmic variance corrections into account, as we describe below.

\subsection{Survey volume}

The RTF has a characteristic phase effect, so the passband of each pseudo-spectrum is blueshifted (and slightly narrowed) when the source is further from the optical centre of the image (see OTELO-I for details). Therefore, each source in our sample has been observed at a different redshift depending on its radial distance to the optical centre of the RTF. Taking this phase effect into account, the range of co-moving volumes is 5130 to $5240 \mathrm{Mpc}^{3}$. Only as a reference, the characteristic co-moving volume of the sample is $5190 \mathrm{Mpc}^{3}$ (i.e. the volume corresponding to a radius that separates the field into two equal areas).

\subsection{Completeness correction}

One of the main challenges when deriving the LF is to estimate (and correct) the incompleteness of the sample. We performed the simulations of objects' detectability in the pseudo-spectra dominium (flux-continuum and flux-observed line equivalent width), instead of the common approach of injecting synthetic sources on the real background. In our methodology, a particular simulation is composed of one synthetic pseudo-spectrum for each node of the simulation grid in the Full Width at Half Maximum FWHM-continuum-amplitude space. The synthetic pseudospectra are affected by random sky plus photon noise components scaled to the noise distribution of each image (slice) of the OTELO tomography. Each spectrum was then convolved by the instrumental response of the tunable filter scan to obtain the simulated pseudo-spectra. The parameters were sampled in larger ranges than those covered in the distributions observed in the real OTELO data. With respect to the noise distribution of pseudospectra, we obtained it by sampling the effective OTELO field in each slice using regions of $1.27 \mathrm{arcsec}^{2}$. This area corresponds to the mode of the distribution of the effective size of the OTELO sources and it is close to 2 times the area of a point source in OTELO. This methodology is fully described in RamónPérez et al. (2019a) and Bongiovanni et al. (2020). This type of data set was fitted by a sigmoid algebraic function (similar in behaviour to e.g. the error function, erf) of the form:

$d=\frac{a F}{\sqrt{c+F^{2}}}$,

where $F=\log \left(f_{1}\right)+b$, with $f_{1}$ being the line flux. We assume $a=0.972 \pm 0.007, b=18.373 \pm 0.092$, and $c=0.475 \pm 0.122$, as obtained in Bongiovanni et al. (2020) (see Figure 4 in that work). This function constitutes the basis of the LF completeness correction. We note that due to the similarity of the redshifts ranges for $\mathrm{H} \beta$ and [O III] samples in the OTELO survey, we adopted the same function as for [O III] OTELO emitters .

\subsection{Cosmic variance}

Since the OTELO survey covers a small sky area at about $0.015 \mathrm{deg}^{2}$, the effects of the cosmic variance (CV) are remarkable (Stroe \& Sobral 2015; Ramón-Pérez et al. 2019a), 
Table 1. Binned values of the observed $\mathrm{H} \beta$ luminosity function. Errors in column 2 include all uncertainties described in Section 4. The fourth column contains the observed number (i.e. before completeness correction) of the $\mathrm{H} \beta$ ELSs in each luminosity bin and the last one shows the cosmic variance parametrisation per bin using the prescription by Somerville et al. (2004).

\begin{tabular}{ccccc}
\hline $\begin{array}{c}\log L(\mathrm{H} \beta) \\
{\left[\mathrm{erg} \mathrm{s}^{-1}\right]}\end{array}$ & $\begin{array}{c}\log \phi \\
{\left[\mathrm{Mpc}^{-3} \mathrm{dex}^{-1}\right]}\end{array}$ & $\begin{array}{c}\text { Number of } \\
\mathrm{H} \beta\end{array}$ & $\begin{array}{c}\text { Typical } \\
\text { Completeness }\end{array}$ & $\sigma_{\mathrm{CV}}$ \\
\hline & & 8 & 0.63 & 0.51 \\
39.24 & $-2.11_{-0.43}^{+0.21}$ & 12 & 0.78 & 0.54 \\
39.56 & $-2.04_{-0.41}^{+0.21}$ & 10 & 0.84 & 0.57 \\
39.88 & $-2.14_{-0.46}^{+0.22}$ & 3 & 0.88 & 0.61 \\
40.20 & $-2.69_{-0.79}^{+0.26}$ & 4 & 0.91 & 0.70 \\
40.52 & $-2.58_{-0.85}^{+0.27}$ & 3 & 0.93 & 0.75 \\
41.84 & $-2.71_{-1.25}^{+0.29}$ & & & \\
\hline
\end{tabular}

especially when compared with surveys with larger volumes (see table 2). It is then clear that obtaining an estimation for the CV effects is essential when characterising the $\mathrm{H} \beta$ luminosity function.

To obtain an uncertainty value due to the $\mathrm{CV}\left(\sigma_{\mathrm{CV}}\right)$ for the science case addressed in this work, we firstly tested the approach given in Bongiovanni et al. (2020), who followed the prescription of Moster et al. (2011). This approach is based on predictions from cold dark matter theory and the galaxy bias, and it takes into account the surveyed area and the redshift range sampled as input values. The mean $\mathrm{CV}$ uncertainty obtained for our $\mathrm{H} \beta$ sample is close to 0.4 . But the mean density of our line emitters at $z=0.9$ is about $1.2 \pm 0.8 \times 10^{-2} \mathrm{Mpc}^{-3}$, which means that the expected $\mathrm{CV}$ effects could be even larger and markedly dependant on the $\mathrm{H} \beta$ luminosity. Hence, we examined the $\mathrm{CV}$ estimations using the recipe provided by Somerville et al. (2004), which is based on number densities against average redshifts in deep surveys, independently of the clustering strength. We estimated this uncertainty for six luminosity bins. The mean CV value obtained using this estimation is $\sigma_{\mathrm{CV}}=0.61$. Noticing the differences between these two estimations, we adopted the Somerville et al. (2004) prescription since it is more conservative. Table 1 shows the CV estimation for each luminosity bin. As stressed in Section 4.4 , the uncertainty due to the $\mathrm{CV}$ is the greatest contributor to the overall uncertainty of the LF.

\subsection{The luminosity function}

The OTELO survey was mainly designed to obtain a large database of emission-line objects at different epochs. The volume at redshift $z \sim 0.9$ stands out in terms of the number of raw ELS candidates obtained, as shown in OTELOI $(\mathrm{H} \beta$ and [O III] emitters given the wavelength range covered).

The survey produced an unprecedented sampling of the faint end of the $\mathrm{H} \beta /[\mathrm{O} \mathrm{III}]-\mathrm{LF}$ due to its low limiting flux, which was obtained by staring at a narrow region of the sky with long exposure times. However, this observational strategy hinders the capability of tracing the bright end, precisely because the small angular size covered $\left(\sim 0.015 \mathrm{deg}^{2}\right)$ decreases the chances of detecting high luminosity galaxies.
We computed the luminosity for each galaxy of our ELS sample from the fluxes obtained from inverse deconvolution. The $\mathrm{H} \beta$ luminosity is distributed in the range $39.07<\log L(\mathrm{H} \beta)<40.98$. Then we computed the number $\Phi$ of galaxies per unit volume $(\mathrm{V})$ and per unit $\mathrm{H} \beta$ -luminosity $\log L(\mathrm{H} \beta)$. This number is provided by:

$$
\Phi[\log L(\mathrm{H} \beta)]=\kappa \frac{4 \pi}{\Omega} \sum_{i} \frac{1}{d_{i}},
$$

where $d_{i}$ is the detection probability defined above for $i$ galaxies, $\Omega$ is the surveyed solid angle $\left(\sim 4.7 \times 10^{-6} \mathrm{str}\right)$, and $\kappa$ is a normalisation factor proportional to $V_{\text {max }}^{-1}$, which is the volume limited by redshifted $\mathrm{H} \beta$ at the maximal spectral range covered by the OTELO scan, including the effect of the wavelength variation with the distance to the optical centre mentioned above.

The Schechter function (Schechter 1976) is the formalism adopted to describe the luminosity function, which is defined as follows:

\section{$\Phi[\log L(\mathrm{H} \beta)] \mathrm{d} \log L=\phi(L) \mathrm{d} L$}

where $\phi(L) \mathrm{d} L \equiv \phi^{*}\left(L / L^{*}\right)^{\alpha} \exp \left(-L / L^{*}\right) \mathrm{d}\left(L / L^{*}\right)$. The parameters $L^{*}, \phi^{*}$, and $\alpha$ are the characteristic value that separates the high and low luminosity regimes in the LF, the number density at $L^{*}$, and the slope of the faint end of the function, respectively.

A Schechter function was fitted to the completeness corrected data given in Table 1, using a least-squares minimisation algorithm based on the Levenberg-Marquardt method. The uncertainties pertaining to the number density include all the corrections mentioned in this section, including the Poisson error. However, it is worth mentioning that the cosmic variance uncertainty is the main contributor in every bin. The parameters obtained from the completenesscorrection LF from OTELO are summarised in Table 2.

\section{Discussion}

As we have mentioned previously, OTELO is an ultra-deep pencil-beam survey. OTELO reaches emission-line fluxes as faint as $10^{-19} \mathrm{erg} \mathrm{s}^{-1} \mathrm{~cm}^{-2}$, but it covers a field of view of about $56 \operatorname{arcmin}^{2}$. This characteristic determines the kind of galaxies that constitute the different populations detected by the survey. The total population is composed of several disconnected emission-line populations at different redshift intervals, selected by the presence of independent emission lines at the corresponding redshift.

In this way, at each redshift we are covering a given volume that is smaller than the typical volumes enclosed by surveys that cover wider apparent fields. The range of the LF that is best traced by OTELO always corresponds to low luminosities, that is, the range of the LF characterised by the exponential slope $\alpha$. This is complementary to regular surveys where this parameter is the most poorly determined. This is one of the main reasons for the extra value of ultra-deep surveys following the OTELO approach.

On the other hand, OTELO covers a relatively small volume of the Universe at $z=0.9$ when compared with previous surveys (see Villar et al. 2008; Sobral et al. 2015). For this reason, the probability of detecting luminous sources is 
Table 2. Best-fit Schechter parameters of OTELO LF for the H $\beta$ ELS samples and its integrals.

\begin{tabular}{cccccccc}
\hline $\begin{array}{c}\text { Line and } \\
\text { dataset }\end{array}$ & $\begin{array}{c}\text { Number } \\
\text { of sources }\end{array}$ & $\begin{array}{c}\text { Redshift } \\
\text { Range }\end{array}$ & $\begin{array}{c}\mathrm{V}_{c}(a) \\
{\left[\mathrm{Mpc}^{3}\right]}\end{array}$ & $\begin{array}{c}\log \phi^{*} \\
{\left[\mathrm{Mpc}^{-3}\right]}\end{array}$ & $\begin{array}{c}\operatorname{logL} L^{*} \\
{\left[\mathrm{erg} \mathrm{s}^{-1}\right]}\end{array}$ & $\begin{array}{c}\operatorname{logL} \text { range } \\
{\left[\mathrm{erg} \mathrm{s}^{-1}\right]}\end{array}$ \\
\hline \hline H $\beta$ OTELO & 40 & $0.86-0.90$ & 5190 & $-3.08 \pm 0.19$ & $41.34($ fixed $)$ & $-1.36 \pm 0.15$ & $39.32-41.31$ \\
$\mathrm{H} \beta$ OTELO+C ${ }^{(b)}$ & 739 & $0.78-0.90$ & $\sim 10^{6}$ & $-3.40_{-0.23}^{+0.20}$ & $41.65_{-0.09}^{+0.11}$ & $-1.43 \pm 0.12$ & $39.08-42.5$ \\
$\mathrm{H} \beta+\left[\right.$ O III] K ${ }^{(c)}$ & 1669 & $0.83-0.85$ & $1.79 \times 10^{5}$ & $-2.55_{-0.03}^{+0.04}$ & $41.79_{-0.05}^{+0.03}$ & $-1.6($ fixed $)$ & $41.0-42.6$ \\
{$[$ O III] OTELO } & 184 & $0.78-0.87$ & $6.6 \times 10^{3}$ & $-2.10 \pm 0.11$ & $41.46 \pm 0.09$ & $-1.03 \pm 0.08$ & $39.2-42.0$ \\
\hline
\end{tabular}

Notes.

${ }^{(a)}$ Co-moving volume. ${ }^{(b)}$ Comparat et al. (2016) ${ }^{(c)}$ Khostovan et al. (2015)

small and most of the $\mathrm{H} \beta$ emitters detected correspond to low $\mathrm{H} \beta$ luminosities, which translates into sub- $L^{*}$ galaxies, most of them being dwarf systems. The low $\mathrm{H} \beta$ luminosity could also correspond to star-forming processes in the final phase, but the short duration of this step implies a low probability of being detected in such a specific evolutive stage.

\subsection{SFR properties}

At $z \sim 0.9$, conventional surveys mainly trace the overall population of disc galaxies in an enhanced star-formation phase that is reflected in cosmic star formation history of the Universe (see, e.g. Villar et al. (2011), Madau \& Dickinson (2014)). Most of these $L^{*}$ systems are easy to detect in the NIR, with a fraction of the population at $z \sim 0.9$ qualifying as luminous infrared galaxies (LIRGs, with stellar masses approximately of $10^{11} \mathrm{M}_{\odot}$ ) and UV-bright systems. On the contrary, OTELO is tracing a population more similar to late-type and dwarf star-forming galaxies in the Local Universe (i.e. the Magellanic Clouds). This is shown in Figs. 2 and 4 where the peak of observed galaxies is around $\log M_{*}\left[\mathrm{M}_{\odot}\right] \sim 8.5$. The less massive the galaxies, the larger the uncertainties are in mass. Among those objects that could be assigned a morphological type, most were classified as disc and spiral, and they present masses and sSFRs appear in the medium-high range.

Figure 5 shows the stellar mass as a function of the $\mathrm{SFR}$ and sSFR in the $\mathrm{H} \beta$ OTELO sample compared with the local number density distribution of SF galaxies in the SDSS database with $0.02<z<0.085$, as obtained by Renzini \& Peng (2015). This figure clearly shows that the $\mathrm{H} \beta$ OTELO sample at $z \sim 0.9$ (i.e. a universe age of $6.3 \mathrm{Gyr}$ ) shares its position with the SF main sequence (hereafter SF-MS) for local galaxies with the exception of one more massive $\log M_{*}>10$ located in the green valley (the one with $\log \mathrm{s} S F R \sim 11.2$ ). It suggests that there is no redshift evolution of the SF-MS in the low-mass regime, in contrast with the observed evolution of the SF-MS for masses with $\log M_{*}>9$ (e.g. Popesso et al. 2019, and references therein). As a comparison, in bottom left panel of Fig. 5, we plotted SF-MS at $z$ around 0.8 as defined by several authors extrapolated to our observed mass range. ${ }^{3}$ In the general sce-

${ }^{3}$ In Speagle et al. (2014); Schreiber et al. (2015) and Santini
et al. (2015), the samples are composed of galaxies with masses
larger than $10^{9.5} \mathrm{M} \odot$. The results of Whitaker et al. (2014) are
based on galaxies with masses larger than $10^{9} \mathrm{M} \odot$, but assuming
that the correction in stellar masses is only right for values larger Article number, page 8 of 13 nario of observed downsizing, massive galaxies form most of their stars earlier and on shorter timescales, while less massive galaxies evolve on longer timescales (Cowie et al. 1996). Low-mass star-forming galaxies at $z=0.9$ detected by OTELO present similar properties as low-mass starforming galaxies in the Local Universe, suggesting that the low mass population of star-forming galaxies is present all along the Universe epochs, with no signs of a favorite epoch of formation or star formation enhancement from $z=1$ to now. It is worth mentioning that most of classic surveys for star-forming galaxies do not properly trace low luminosity star-forming systems. This is reflected in the luminosity function.

\subsection{Luminosity function}

The OTELO $\mathrm{H} \beta$ LF best-fit values are shown in Table 2 and Figure 6. As shown there, we performed two different $\mathrm{H} \beta$ LF fittings.

The first fit was constructed using the dust-corrected $L(\mathrm{H} \beta)$ values. In this case, the $\log L^{*}$ parameter was fixed to a constant value of 41.34 since our data cover the fainter end of the LF and hence there are not enough to constraint the high and low regimes boundary. This value was drawn from looking at past work from previous narrow-band studies, specifically as a mean value from those used by Comparat et al. (2016) and Khostovan et al. (2015) (see Table 2). The values obtained for the $\log \phi^{*}$ and $\alpha$ parameters are -3.08 and -1.36 , respectively. As we have explained above, this fitting takes advantage of dust-corrected luminosity values so a comparison with previous works would not be interesting since those used non-corrected data. However, the value obtained for the slope of the faint end, $\alpha$, is very significant since our sample extends further than any other previous work and it would constitute a solid and unpredecented $\alpha$ for dust-corrected $\mathrm{H} \beta \mathrm{LF}$ at $z \sim 0.9$. This fitting is shown in the first panel of Figure 6 . In Table 2 we have also included the parameters of the OTELO [O III] LF from Bongiovanni et al. (2020) at a similar redshift range as our own.

than $10^{10} \mathrm{M} \odot$. Our galaxies are below the extrapolated position of the low mass SF-MS at $z \sim 0.9$ even taking into account an intrinsic scatter of the SF-MS of about 0.3 dex (e.g. Kurczynski et al. 2016). As an additional test, we computed the sSFR increasing the extinction by a factor of 3 (i.e. to mimic a case where $\left.E(B-V)_{\text {Balmer }}=3 \times E(B-V)_{\mathrm{SED}}\right)$. In this case, 12 galaxies are above the extrapolation of Schreiber et al. (2015) SF-MS, and 28 are below. Actually, a decrease in the Schreiber et al. (2015) SF-MS of 0.2 dex would be required for it to be at the median value of the sample (included the extra extinction correction). 

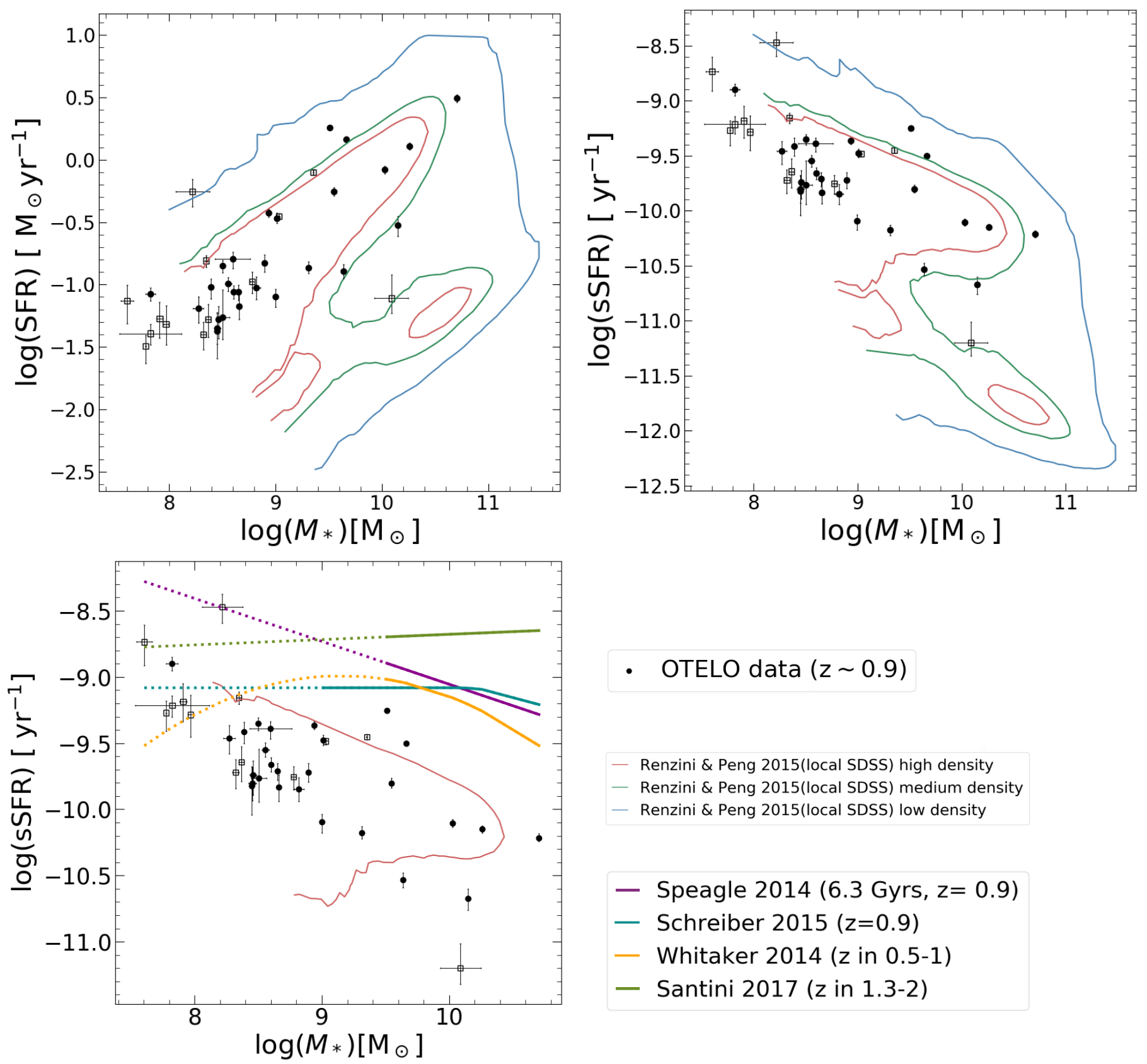

- OTELO data $(z \sim 0.9)$

- Renzini \& Peng 2015(local SDSS) high density

- Renzini \& Peng 2015(local SDSS) medium density

- Renzini \& Peng 2015(local SDSS) low density

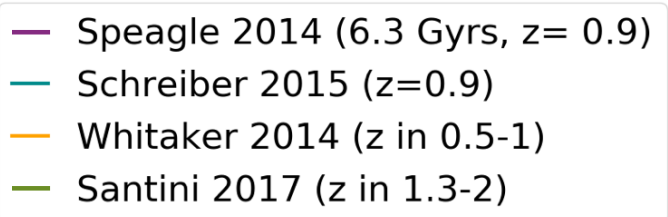

Fig. 5. Stellar masses as a function of SFR (top-left) and sSFR (top-right) for the $\mathrm{H} \beta$ sample. Disc and spheroid galaxies are indicated with empty yellow squares and empty grey circles, respectively. Contours correspond to the number density of galaxies from the SDSS database and obtained by Renzini \& Peng (2015) at values of $1.2 \times 10^{5}$ (red), $7.0 \times 10^{4}$ (green), and $2.0 \times 10^{4}$ (blue), clearly showing the position of the SF main sequence for local galaxies. We differentiate the robust sub-sample of 27 galaxies from the remaining 13 with black circles and empty black squares, respectively. The bottom-left plot clearly shows the local SF main sequence location (the red contour), where most of the galaxies in our sample are located. The plot also shows the different position of the SF main sequence given by different authors. For each MS, we differentiate the mass range used by each author from the extrapolated one by us by plotting the latter with a discontinuous line.

In an attempt to execute a LF fitting where every parameter was set free and, at the same time, to extend our sample to the brighter end, we performed a second $\mathrm{H} \beta \mathrm{LF}$ fitting joining the OTELO data with $\mathrm{H} \beta$ at $z \sim 0.8$ data from Comparat et al. (2016). In this case, we used the non dustcorrected $\mathrm{L}(\mathrm{H} \beta)$ values, since those from Comparat et al. (2016) were not corrected from dust extinction as well. The second panel of Figure 6 portrays this LF fitting as well as the fit from Khostovan. The sample from Khostovan et al.
(2015) contains data from $\mathrm{H} \beta+[\mathrm{O}$ III $] \lambda 4959,5007$ ELS at $z \sim 0.8$.

The largest relative uncertainties on the Schechter parameters obtained after the non-linear fitting of both LFs correspond to the $68.27 \%$ confidence interval of each parameter. The inset in Figure 5 shows the strong correlation between $\phi *$ and $\alpha$ parameters.

As stated above, Schechter-LFs parameters are more or less correlated, hence the difficulty when trying to compare different fittings. However, a general agreement is observed 


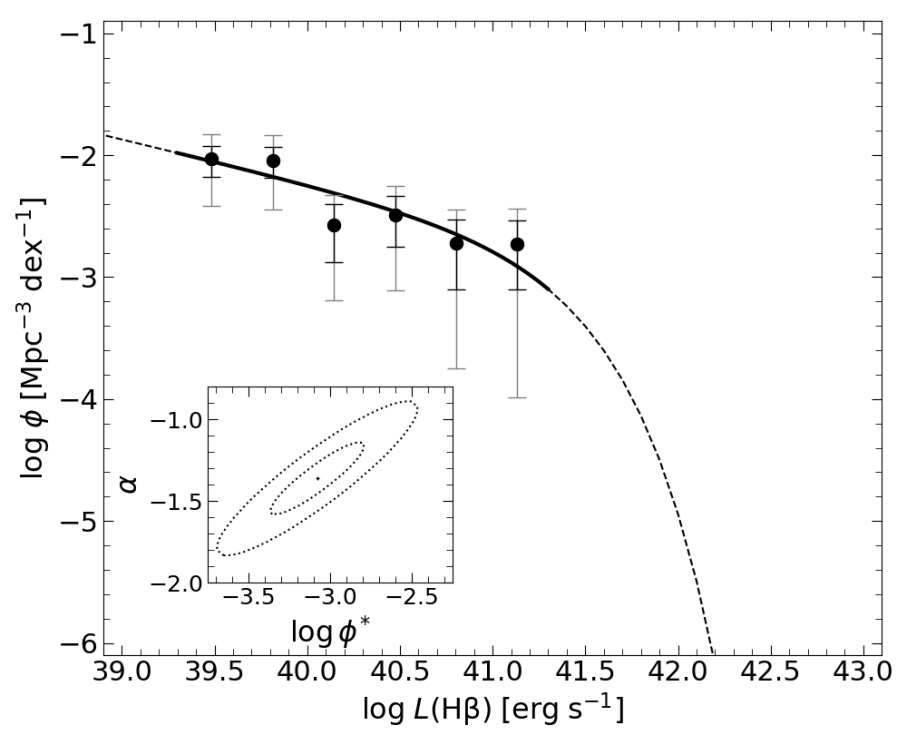

(a)

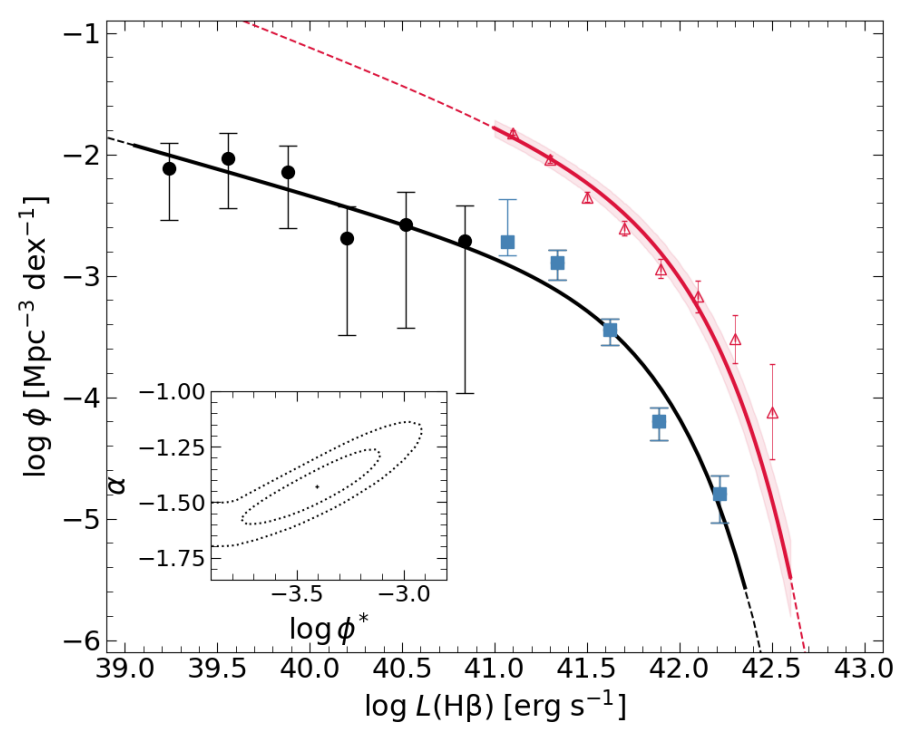

(b)

Fig. 6. Top figure: Completeness and dust extinction-corrected $\mathrm{H} \beta \mathrm{LF}$ at $z \sim 0.9$. The black dots represent the $\mathrm{H} \beta$ sample and the black line shows the fitting of this sample. Shorter error bars represent the Poissonian error and the larger error bars have the rest of the uncertainties evenly added in quadrature (see text for details). Bottom figure: OTELO $\mathrm{H} \beta$ sample (black dots) complemented with high-luminosity data from Comparat et al. (2016) $\mathrm{H} \beta$ sample at $\mathrm{z} \sim 0.8$ (blue squares) fitted LF. Red triangles and the red line represent the literature data from the $\mathrm{H} \beta+[\mathrm{O}$ III $] \lambda 4959,5007$ sample by Khostovan et al. (2015) at $z \sim$ 0.8 and its LF fitting, respectively.

between the LF estimates from previous studies and ours. Figure 6 seemingly shows a significant difference among these different works along the whole LF. In particular, the OTELO $\mathrm{LF}(\mathrm{H} \beta)$ prediction around the surroundings of $L^{*}$ is about $\sim 1$ dex smaller than the model of Khostovan et al. (2015). Similarly, all of the fitted $\phi^{*}$ values from the literature are larger than those from our sample. This is likely because the sample of Khostovan et al. (2015) gathers both [O III] and $\mathrm{H} \beta$ emission lines and, as they predict in their study, the bright end is usually dominated by [O III] emit- ters. In Bongiovanni et al. (2020), we obtain the LF of [O III] at $z \sim 0.8$, getting significantly different values for $\phi^{*}$ and $\alpha$ (see Table 2). This implies that the contribution of the oxygen lines to the LF adds different physical parameters to those influencing the $\mathrm{H} \beta$ emission. The study presented in this paper shows the $\operatorname{LF}(\mathrm{H} \beta)$ based on the fluxes of this line only, without contamination from [O III].

At low luminosities, the LF obtained from the OTELO $\mathrm{H} \beta$ sample extends about 100 times fainter than the most sensitive extreme observed to date. Hence we are observing faint galaxies that other surveys do not detect. Regarding our $\alpha$ value, it slightly differs from the one used by Khostovan et al. (2015) and by Comparat et al. (2016), which are $\sim-1.66$ and $\sim-1.51$, respectively. It must be noted, nonetheless, that the $\alpha$ adopted in Khostovan et al. (2015) is a fixed value derived from previous works that do not reach luminosities as low as the $\mathrm{H} \beta$ OTELO sample does. Hence our results provide a better approach to this estimation. Other studies using $\mathrm{H} \alpha$ at $z=0.8$ (Sobral et al. 2013) reach only $\log L \sim 41.5$, and the values of $\alpha$ reported in those works are based in the bright end of the luminosity function. Here we report the LF estimate based in sources up to $\log L \sim 39.5$.

Moreover, Drake et al. (2013) infer that the detection fraction of ELSs strongly determines the faint-end slope of the LF, but also that the $\alpha$ value is sensitive to the adopted limit of EW of a typical NB survey. Accordingly, since the EW lower limit of OTELO data is around $6 \AA$, we can conclude the faint-end slope value provided by our $\mathrm{H} \beta$ fit is robust enough.

\section{Summary and conclusions}

OTELO is a 2D spectroscopic blind survey, with a spectral resolution of $\mathrm{R}=700$, covering a field in the EGS of $7.5 \times 7.4$ $\operatorname{arcmin}^{2}$ area. Using the OSIRIS TF, a window of $230 \AA$ centred at $9175 \AA$ was scanned with 36 slices evenly spaced by $6 \AA$. OTELO obtained photometric data at consecutive and overlapping wavelength ranges (pseudo-spectra) of all ELS in the field, hence covering a wide range of volumes between $z=0.4$ and 6 . The final product is a set of astrometrycorrected and flux-calibrated images of each slice as well as a pseudo-spectrum for every source of the field, obtained by doing aperture photometry in the images. Details on the survey strategy, data reduction, and main products are provided in OTELO-I.

In this paper, we have exploited the scientific potential of the selection of ELSs detailed in OTELO-I and focused on $\mathrm{H} \beta$ emitters. The selection procedure for this very sample provided 87 preliminary ELSs in the redshift window around $z=0.88$. From this selection, 41 objects constitute the final sample. We performed a deconvolution of their emission lines in order to obtain accurate redshifts, line fluxes, and observed EW. The $\mathrm{H} \beta$ sample is distributed in a redshift range between 0.86 and 0.9 , with a limiting line flux of $\sim 1 \times 10^{-18} \mathrm{erg} \mathrm{s}^{-1} \mathrm{~cm}^{2}$ with an EW as low as $\sim 9 \AA$. Most of the morphologically classified $\mathrm{H} \beta$ ELSs are disc-like galaxies $(76 \%)$, and stellar masses range between $10^{7.6}-10^{10.7} \mathrm{M}_{\odot}$, with $90 \%$ of the sample in the low-mass galaxy population $\left(\mathrm{M}_{*}<10^{10} \mathrm{M}_{\odot}\right)$. After searching for AGN host candidates as described in Sect.2.3, only one source was classified as such. The OTELO survey hence provides 
high sensitivity to the detection of faint SFGs and a very significant minimum line flux.

The SFR was derived from dust and stellar absorptioncorrected $\mathrm{H} \beta$ luminosity. The SFR result places our sample in the SFR main sequence according to Santini et al.(2017), and it induces a similarity between this sources and dwarf star-forming galaxies in the Local Universe. Being our sample mostly formed by galaxies with masses below $10^{9.5} \mathrm{M}_{\odot}$ and under the assumption of a moderate correction of $E(B-V)_{\text {Balmer }} \leq 3 \times E(B-V)_{\mathrm{SED}}$, we conclude that our data are compatible with no evolution in the SFR of low-mass galaxies.

We computed the luminosity for each galaxy of our ELS sample from the fluxes obtained from inverse deconvolution. In sampling a co-moving volume of $\sim 5190 \mathrm{Mpc}^{3}$ and mainly taking the sources of uncertainties into account (primarily CV effects), we obtained the observed non dust-corrected $\mathrm{LF}$ of the $\mathrm{H} \beta$ sample. The sample produced an unprecedented sampling of the faint end of the $\mathrm{H} \beta \mathrm{LF}$ as it is 100 times fainter than the extreme reached by other surveys to date. This dust-corrected OTELO H $\beta$ LF delivered the following Schechter parameters: $\log \phi^{*}=-3.08 \pm 0.19$, $\log L^{*}=41.34$, and $\alpha=-1.36 \pm 0.15$. A second LF fitting was made by extending the bright end of our non dust-corrected sample with the $\mathrm{H} \beta+[\mathrm{O}$ III $] \lambda 4959,5007$ data from Comparat et al. (2016) and the parameters for the best fit for this junction were: $\log \phi^{*}=-3.40 \pm 0.2, \log L^{*}=41.65 \pm 0.1$, and $\alpha=-1.43 \pm 0.12$. This faint-end slope value is consistent with previous similar works, and it represents the most robust alpha estimation at $z \sim 0.8$ published so far, based on the faintest isolated $\mathrm{H} \beta$ (and $\mathrm{H} \alpha$ ) detections to date. In this sense, OTELO is complementary to other surveys, but it provides an unreached sensitivity to smaller, faint galaxies.

Acknowledgements. This paper is dedicated to the memory of our dear friend and colleague Hector Castañeda, unfortunately died on 19 Nov 2020. This work was supported by the Spanish Ministry of Economy and Competitiveness (MINECO) under the grants AYA2014 - 58861 - C3 - 1 - P, AYA2014- 58861 - C3 - 2 - P, AYA2014 58861 - C3 - 3 - P, AYA2013 - 46724-P, AYA2017-88007-C3-1-P, AYA2017-88007-C3-2, MDM-2017-0737 (Unidad de Excelencia María de Maeztu, CAB). APG acknowledge support from ESA through the Faculty of the European Space Astronomy Centre (ESAC) - Funding reference ESAC_549/2019. Based on observations made with the Gran Telescopio Canarias (GTC), installed in the Spanish Observatorio del Roque de los Muchachos of the Instituto de Astrofísica de Canarias, in the island of La Palma. This study makes use of data from AEGIS, a multiwavelength sky survey conducted with the Chandra, GALEX, Hubble, Keck, CFHT, MMT, Subaru, Palomar, Spitzer, VLA, and other telescopes and supported in part by the NSF, NASA, and the STFC. Based on observations obtained with MegaPrime/MegaCam, a joint project of CFHT and CEA/IRFU, at the Canada-France-Hawaii Telescope (CFHT) which is operated by the National Research Council (NRC) of Canada, the Institut National des Science de l'Univers of the Centre National de la Recherche Scientifique (CNRS) of France, and the University of Hawaii. This work is based in part on data products produced at Terapix available at the Canadian Astronomy Data Centre as part of the CanadaFrance-Hawaii Telescope Legacy Survey, a collaborative project of NRC and CNRS. Based on observations obtained with WIRCam, a joint project of CFHT,Taiwan, Korea, Canada, France, at the CanadaFrance-Hawaii Telescope (CFHT) which is operated by the National Research Council (NRC) of Canada, the Institute National des Sciences de l'Univers of the Centre National de la Recherche Scientifique of France, and the University of Hawaii. This work is based in part on data products produced at TERAPIX, the WIRDS (WIRcam Deep Survey) consortium, and the Canadian Astronomy Data Centre. This research was supported by a grant from the Agence Nationale de la Recherche ANR-07-BLAN-0228

\section{References}

Alvarez, P., Rodríguez Espinosa, J. M., \& Sánchez, F. 1998, New A Rev., 42, 553

Arnouts, S., Cristiani, S., Moscardini, L., et al. 1999, MNRAS, 310, 540

Bluck, A. F. L., Mendel, J. T., Ellison, S. L., et al. 2014, MNRAS, 441,599

Bongiorno, A., Mignoli, M., Zamorani, G., et al. 2010, A\&A, 510, A56 Bongiovanni, Á., Ramón-Pérez, M., Pérez García, A. M., et al. 2019, A\&A, 631, A9

Bongiovanni, Á., Ramón-Pérez, M., Pérez García, A. M., et al. 2020, A\&A, 635, A35

Bouché, N., Dekel, A., Genzel, R., et al. 2010, ApJ, 718, 1001

Cardelli, J. A., Clayton, G. C., \& Mathis, J. S. 1989, ApJ, 345, 245

Cepa, J., Alfaro, E. J., Bland-Hawthorn, J., et al. 2003, in Revista Mexicana de Astronomia y Astrofisica Conference Series, Vol. 16, Revista Mexicana de Astronomia y Astrofisica Conference Series, ed. J. M. Rodriguez Espinoza, F. Garzon Lopez, \& V. Melo Martin, 64-68

Cerviño, M., Bongiovanni, A., \& Hidalgo, S. 2016, A\&A, 589, A108 Chiang, C.-Y., Goto, T., Hashimoto, T., et al. 2019, PASJ, 71, 31

Coleman, G. D., Wu, C. C., \& Weedman, D. W. 1980, ApJS, 43, 393

Comparat, J., Zhu, G., Gonzalez-Perez, V., et al. 2016, MNRAS, 461, 1076

Coughlin, A., Rhoads, J. E., Malhotra, S., et al. 2018, ApJ, 858, 96

Cowie, L. L., Songaila, A., Hu, E. M., \& Cohen, J. G. 1996, AJ, 112, 839

Donley, J. L., Koekemoer, A. M., Brusa, M., et al. 2012, ApJ, 748, 142

Drake, A. B., Simpson, C., Collins, C. A., et al. 2013, MNRAS, 433, 796

Driver, S. P., Norberg, P., Baldry, I. K., et al. 2009, Astronomy and Geophysics, 50, 5.12

Elbaz, D., Daddi, E., Le Borgne, D., et al. 2007, A\&A, 468, 33

Elmegreen, D. M., Elmegreen, B. G., Ravindranath, S., \& Coe, D. A. 2007, ApJ, 658, 763

Fujita, S. S., Ajiki, M., Shioya, Y., et al. 2003, ApJ, 586, L115

Gallego, J., Zamorano, J., Aragon-Salamanca, A., \& Rego, M. 1995, ApJ, 455, L1

Geach, J. E., Smail, I., Best, P. N., et al. 2008, MNRAS, 388, 1473

Hayashi, M., Tanaka, M., Shimakawa, R., et al. 2018, PASJ, 70, S17

Hopkins, A. M., Driver, S. P., Brough, S., et al. 2013, MNRAS, 430, 2047

Hopkins, A. M., Miller, C. J., Nichol, R. C., et al. 2003, ApJ, 599, 971 Ilbert, O., Arnouts, S., McCracken, H. J., et al. 2006, A\&A, 457, 841 Kartaltepe, J. S., Mozena, M., Kocevski, D., et al. 2015, ApJS, 221, 11

Kennicutt, Robert C., J. 1998, ARA\&A, 36, 189

Kennicutt, R. C. \& Evans, N. J. 2012, ARA\&A, 50, 531

Khostovan, A. A., Sobral, D., Mobasher, B., et al. 2015, MNRAS, 452, 3948

Kinney, A. L., Calzetti, D., Bohlin, R. C., et al. 1996, ApJ, 467, 38

Kroupa, P. 2001, MNRAS, 322, 231

Kurczynski, P., Gawiser, E., Acquaviva, V., et al. 2016, ApJ, 820, L1

Le Fèvre, O., Cassata, P., Cucciati, O., et al. 2013, A\&A, 559, A14

López-Sanjuan, C., Díaz-García, L. A., Cenarro, A. J., et al. 2019, A\&A, 622, A51

Madau, P. \& Dickinson, M. 2014, ARA\&A, 52, 415

Matthee, J., Sobral, D., Best, P., et al. 2017, MNRAS, 471, 629

Moster, B. P., Somerville, R. S., Newman, J. A., \& Rix, H.-W. 2011, ApJ, 731, 113

Nadolny, J., Bongiovanni, Á., Cepa, J., et al. 2021, A\&A, 647, A89

Nadolny, J., Lara-López, M. A., Cerviño, M., et al. 2020, A\&A, 636, A84

Newman, J. A., Cooper, M. C., Davis, M., et al. 2013, ApJS, 208, 5

Noeske, K. G., Weiner, B. J., Faber, S. M., et al. 2007, ApJ, 660, L43

Pérez-Martínez, R. M. 2016, PhD "From Xrays to Far Infrared: Galaxy Cluster ZwCL0024+1652 under the multiwavelength limelight", Universidad Complutense de Madrid

Popesso, P., Morselli, L., Concas, A., et al. 2019, MNRAS, 490, 5285

Ramón-Pérez, M., Bongiovanni, Á., Pérez García, A. M., et al. 2019a, A\&A, 631, A10

Ramón-Pérez, M., Bongiovanni, Á., Pérez García, A. M., et al. 2019b, A\&A, 631, A11

Renzini, A. \& Peng, Y.-j. 2015, ApJ, 801, L29

Santini, P., Ferguson, H. C., Fontana, A., et al. 2015, ApJ, 801, 97

Schechter, P. 1976, ApJ, 203, 297

Schreiber, C., Pannella, M., Elbaz, D., et al. 2015, A\&A, 575, A74 
Sobral, D., Matthee, J., Best, P. N., et al. 2015, MNRAS, 451, 2303

Sobral, D., Swinbank, A. M., Stott, J. P., et al. 2013, ApJ, 779, 139

Somerville, R. S., Lee, K., Ferguson, H. C., et al. 2004, ApJ, 600, L171

Speagle, J. S., Steinhardt, C. L., Capak, P. L., \& Silverman, J. D. 2014, ApJS, 214, 15

Storey, P. J. \& Hummer, D. G. 1995, MNRAS, 272, 41

Stroe, A. \& Sobral, D. 2015, MNRAS, 453, 242

Villar, V., Gallego, J., Pérez-González, P. G., et al. 2011, ApJ, 740, 47

Villar, V., Gallego, J., Pérez-González, P. G., et al. 2008, ApJ, 677, 169

Villaverde, M., Cerviño, M., \& Luridiana, V. 2010, A\&A, 517, A93

Whitaker, K. E., Franx, M., Leja, J., et al. 2014, ApJ, 795, 104 


\section{Appendix A: Catalogue of $\mathrm{H} \beta$ emitters}

In Table A.1 and A.2, we summarise the main properties of our emitters.

Table A.1. Characteristics of the 27 non-AGN bona fide sources (see Sect. 2.2).

\begin{tabular}{|c|c|c|c|c|c|c|}
\hline ID & $z$ & $\begin{array}{c}\text { Flux } \\
{\left[\times 10^{-17} \mathrm{erg} / \mathrm{s} / \mathrm{cm}^{2}\right]}\end{array}$ & $\begin{array}{l}\mathrm{EW} \\
{[\AA]}\end{array}$ & $\begin{array}{c}\log M \\
{\left[\mathrm{M}_{\odot}\right]}\end{array}$ & $(g-i)$ & $\begin{array}{c}\mathrm{SFR} \\
{\left[\mathrm{M}_{\odot} \mathrm{yr}^{-1}\right]}\end{array}$ \\
\hline 797 & 0.863 & $0.71 \pm 0.1$ & $121.3_{-234}^{+17.3}$ & $8.60 \pm 0.16$ & $0.99 \pm 0.3$ & $0.16_{-0.03}^{+0.02}$ \\
\hline 1223 & 0.862 & $0.15_{-0.05}^{+0.1}$ & $44.71_{-18.9}^{+48.4}$ & $8.50 \pm 0.06$ & $0.54 \pm 0.14$ & $0.06_{-0.02}^{+0.03}$ \\
\hline 1981 & 0.864 & $0.24 \pm 0.06$ & $49.34_{-136}^{+16.93}$ & $8.27 \pm 0.05$ & $0.17 \pm 0.12$ & $0.07 \pm 0.02$ \\
\hline 2130 & 0.867 & $0.44 \pm 0.05$ & $89.5_{-15.9}^{+21.5}$ & $7.82 \pm 0.05$ & $0.03 \pm 0.14$ & $0.08 \pm 0.01$ \\
\hline 2236 & 0.864 & $1.26_{-01}^{+0.1}$ & $48.2_{-5.9}^{+5.9}$ & $9.01 \pm 0.01$ & $0.38 \pm 0.02$ & $0.34 \pm 0.03$ \\
\hline 2304 & 0.901 & $4.59_{-0.3}^{+0.1}$ & $14.63 \pm 1.09$ & $10.71 \pm 0.003$ & $1.13 \pm 0.006$ & $3.10 \pm 0.2$ \\
\hline 2447 & 0.899 & $0.8_{-0.08}^{+0.09}$ & $18.6_{-2.2}^{+2.5}$ & $9.31 \pm 0.006$ & $0.49 \pm 0.02$ & $0.14_{-0.01}^{+0.02}$ \\
\hline 2623 & 0.862 & $0.16 \pm 0.06$ & $24.45_{-9.4}^{+2.2}$ & $8.45 \pm 0.02$ & $0.19 \pm 0.05$ & $0.04 \pm+0.02$ \\
\hline 2644 & 0.891 & $0.52 \pm 0.06$ & $41.97_{-6.2}^{+6.4}$ & $8.6 \pm 0.01$ & $0.37 \pm 0.03$ & $0.09 \pm 0.01$ \\
\hline 2722 & 0.893 & $5.13_{-0.2}^{+0.3}$ & $39.3_{-2.3}^{+2.2^{2}}$ & $9.67 \pm 0.002$ & $0.32 \pm 0.005$ & $1.45 \pm 0.07$ \\
\hline 4971 & 0.885 & $0.63 \pm 0.1$ & $37.51_{-6.74}^{+7.39}$ & $8.90 \pm 0.01$ & $0.55 \pm 0.03$ & $0.15_{-0.02}^{+0.03}$ \\
\hline 5133 & 0.874 & $0.66 \pm 0.1$ & $11.40 \pm 2.08$ & $10.15 \pm 0.02$ & $1.31 \pm 0.03$ & $0.3_{-0.06}^{+0.05}$ \\
\hline 5498 & 0.9 & $1.84 \pm 0.1$ & $88.27_{-8.72}^{+10.28}$ & $8.93 \pm 0.004$ & $0.06 \pm 0.01$ & $0.37 \pm 0.3$ \\
\hline 5808 & 0.878 & $9.23 \pm 0.02$ & $54.74_{-1.92}^{+1.70}$ & $9.51 \pm 0.001$ & $0.23 \pm 0.002$ & $1.81_{-0.04}^{+0.05}$ \\
\hline 5922 & 0.884 & $0.19_{-0.05}^{+0.06}$ & $50.16_{-15.93}^{+2.95}$ & $8.45 \pm 0.03$ & $0.55 \pm 0.07$ & $0.05 \pm 0.01$ \\
\hline 6474 & 0.903 & $0.50 \pm 0.07$ & $55.84_{-9.98}^{+10.34}$ & $8.65 \pm 0.01$ & $0.36 \pm 0.03$ & $0.09 \pm 0.01$ \\
\hline 6890 & 0.862 & $0.33 \pm 0.1$ & $31.72_{-9.91}^{+10.30}$ & $8.46 \pm 0.03$ & $0.35 \pm 0.06$ & $0.05 \pm 0.02$ \\
\hline 7023 & 0.885 & $0.84 \pm 0.09$ & $71.01_{-10.84}^{+12.01}$ & $8.50 \pm 0.02$ & $0.35 \pm 0.05$ & $0.14 \pm 0.2$ \\
\hline 7048 & 0.9 & $0.75_{-0.09}^{+0.10}$ & $19.88_{-2.87}^{+3}$ & $9.64 \pm 0.01$ & $0.75 \pm 0.02$ & $0.13 \pm 0.2$ \\
\hline 7467 & 0.88 & $0.44_{-0.05}^{+0.06}$ & $74.61_{-13.15}^{+17.47}$ & $8.56 \pm 0.03$ & $0.56 \pm 0.06$ & $0.10 \pm 0.01$ \\
\hline 7602 & 0.904 & $3.42 \pm 0.2$ & $14.68_{-1.11}^{+1.03}$ & $10.03 \pm 0.001$ & $0.55 \pm 0.003$ & $0.83 \pm 0.06$ \\
\hline 7629 & 0.876 & $2.40 \pm 0.2$ & $36.4_{-3.27}^{+3.80}$ & $9.55 \pm 0.004$ & $0.55 \pm 0.01$ & $0.56_{-0.04}^{+0.05}$ \\
\hline 7726 & 0.875 & $0.41_{-0.09}^{+0.10}$ & $46.01_{-11.53}^{+1.4 .04}$ & $8.66 \pm 0.02$ & $0.36 \pm 0.04$ & $0.07_{-0.01}^{+0.02}$ \\
\hline 7953 & 0.864 & $0.50 \pm 0.09$ & $64.97_{-12.94}^{+15}$ & $8.39 \pm 0.013$ & $0.11 \pm 0.04$ & $0.09 \pm 0.02$ \\
\hline 8010 & 0.862 & $0.42_{-0.08}^{+0.09}$ & $62.08_{-16.11}^{+17.68}$ & $8.82 \pm 0.04$ & $0.55 \pm 0.09$ & $0.09 \pm 0.02$ \\
\hline 10927 & 0.861 & $0.50_{-0.08}^{+0.08}$ & $28.90_{-566}^{+4.69}$ & $8.99 \pm 0.01$ & $0.36 \pm 0.01$ & $0.08 \pm 0.01$ \\
\hline 11014 & 0.904 & $2.68 \pm 0.2$ & $17.35_{-1.2}^{+1.1}$ & $10.26 \pm 0.002$ & $0.94 \pm 0.004$ & $1.29 \pm 0.08$ \\
\hline
\end{tabular}

Table A.2. Same as Table A.1, but for the 13 sources which cannot been considered as bona fide ones (see Sect. 2.2.).

\begin{tabular}{ccccccc}
\hline ID & $z$ & $\begin{array}{c}\text { Flux } \\
{\left[\times 10^{-17} \mathrm{erg} / \mathrm{s} / \mathrm{cm}^{2}\right]}\end{array}$ & $\begin{array}{c}\mathrm{EW} \\
{[\AA]}\end{array}$ & $\begin{array}{c}\log M \\
{\left[\mathrm{M}_{\odot}\right]}\end{array}$ & $(g-i)$ & $\begin{array}{c}\text { SFR } \\
{\left[\mathrm{M}_{\odot} \mathrm{yr}^{-1}\right]}\end{array}$ \\
\hline 2755 & 0.862 & $0.52 \pm 0.13$ & $47.5_{-12.9}^{+14.3}$ & $8.22 \pm 0.16$ & $0.81 \pm 0.3$ & $0.56 \pm 0.14$ \\
4460 & 0.855 & $0.41_{-0.13}^{+0.22}$ & $72.90_{-22.7}^{+48.9}$ & $10.09 \pm 0.16$ & $1.24 \pm 0.24$ & $0.08_{-0.02}^{+0.04}$ \\
5156 & 0.873 & $0.46 \pm 0.09$ & $30.18_{-6.97}^{+6.82}$ & $8.78 \pm 0.02$ & $0.55 \pm 0.04$ & $0.11 \pm 0.21$ \\
5864 & 0.873 & $0.24 \pm 0.06$ & $39.58_{-10.83}^{+13.13}$ & $8.32 \pm 0.02$ & $0.36 \pm 0.06$ & $0.04 \pm 0.01$ \\
6456 & 0.903 & $3.90 \pm 0.2$ & $56.37_{-2.93}^{+2.83}$ & $9.35 \pm 0.001$ & $0.09 \pm 0.004$ & $0.80 \pm 0.03$ \\
6838 & 0.862 & $0.12 \pm 0.03$ & $81.34_{-28.31}^{+33.87}$ & $7.78 \pm 0.001$ & 0.35 & $0.03 \pm 0.01$ \\
7868 & 0.886 & $0.78_{-0.09}^{+0.08}$ & $142.97_{-27.89}^{+34.03}$ & $8.35 \pm 0.02$ & $0.14 \pm 0.04$ & $0.16 \pm 0.02$ \\
8187 & 0.892 & $1.48_{-0.09}^{+0.08}$ & $80.12_{-7.36}^{+7.20}$ & $9.03 \pm 0.01$ & $0.54 \pm 0.02$ & $0.35 \pm 0.02$ \\
9344 & 0.862 & $0.19_{-0.06}^{+0.07}$ & $37.39_{-14.33}^{+15.22}$ & $7.60 \pm 0.1$ & $0.25 \pm 0.15$ & $0.07 \pm 0.03$ \\
9927 & 0.861 & $0.17_{-0.06}^{+0.07}$ & $39.49_{-13.55}^{+20.30}$ & $7.97 \pm 0.02$ & $0.21 \pm 0.06$ & $0.05 \pm 0.02$ \\
10097 & 0.86 & $0.25 \pm 0.05$ & $77.41_{-18.58}^{+23.22}$ & $7.82 \pm 0.3$ & $0.34 \pm 0.91$ & $0.04 \pm 0.01$ \\
10988 & 0.863 & $0.19_{-0.06}^{+0.07}$ & $52.17_{-2.20}^{+31.32}$ & $7.91 \pm 0.03$ & $0.19 \pm 0.07$ & $0.05 \pm 0.02$ \\
11063 & 0.864 & $0.20 \pm 0.06$ & $41.76_{-13.2}^{+16.2}$ & $8.37 \pm 0.02$ & $0.31 \pm 0.05$ & $0.05 \pm 0.02$ \\
\hline
\end{tabular}

